\title{
Fast and Efficient Fc-Specific Photoaffinity Labeling To Produce Antibody-DNA Conjugates
}

Christiane Stiller ${ }^{\dagger}$, Hooman Aghelpasand ${ }^{\ddagger}$, Tobias Frick $^{\ddagger}$, Kristina Westerlund $^{\dagger}$, Afshin Ahmadian $^{\ddagger}$, Amelie Eriksson Karlström ${ }^{+}$

${ }^{\dagger}$ Department of Protein Science, School of Engineering Sciences in Chemistry, Biotechnology and Health, KTH Royal Institute of Technology, AlbaNova University Center, 10691 Stockholm, Sweden

${ }^{\ddagger}$ Department of Gene Technology, School of Engineering Sciences in Chemistry, Biotechnology and Health, KTH Royal Institute of Technology, Science for Life Laboratory, 17165 Solna, Sweden

\section{Supplementary information}

A
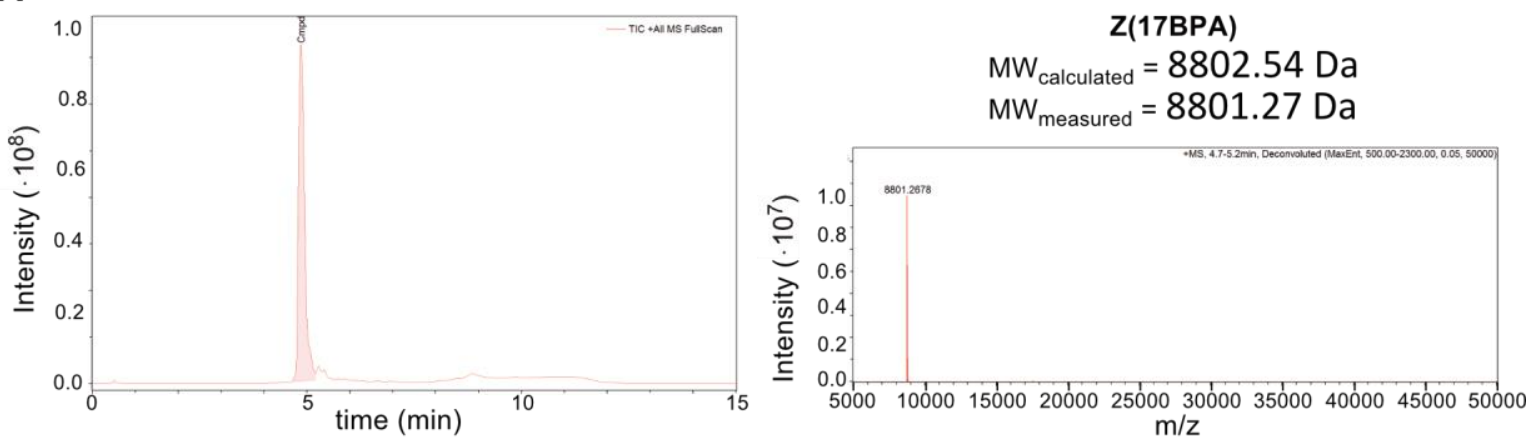

B
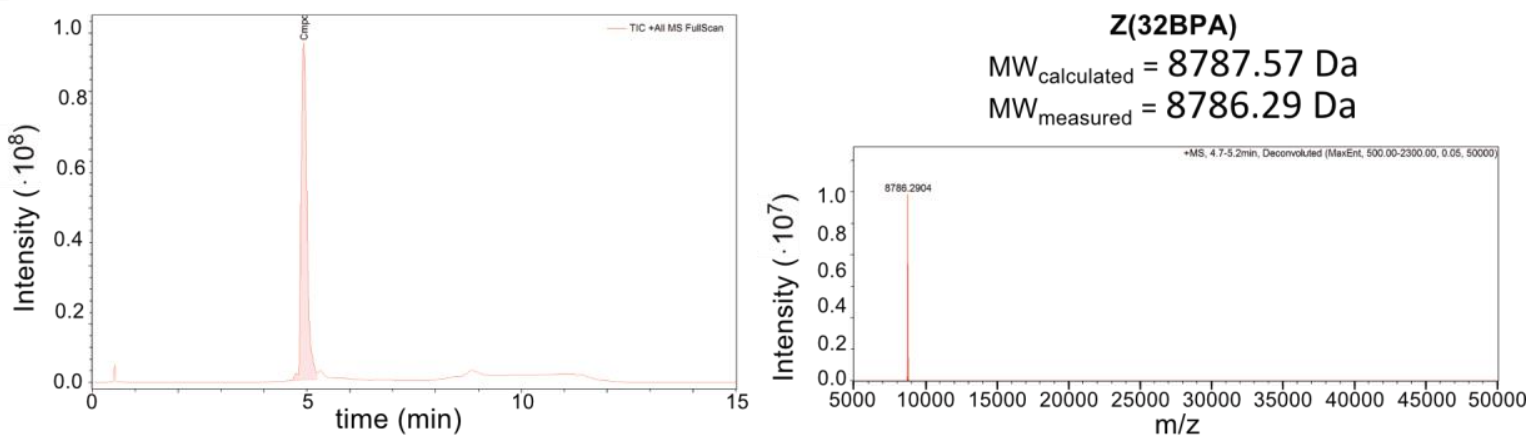

C
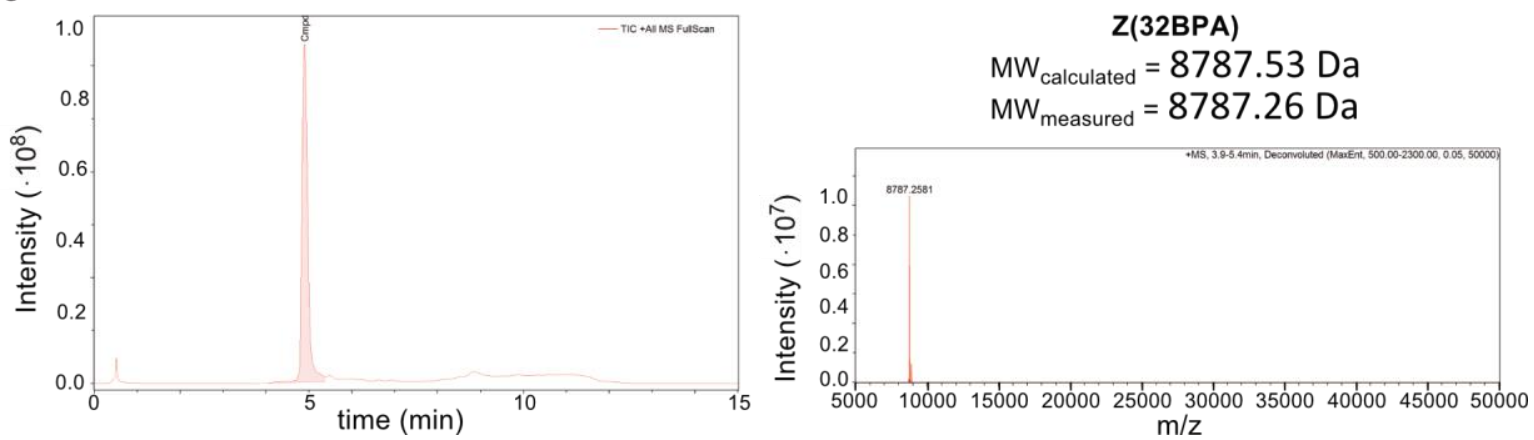

Figure S1: ESI-MS spectra for the three $Z(x B P A)$-domains. For each protein the total ion count is shown on the left side and the deconvoluted spectra of its main peak on the right side. A: analysis of Z(17BPA). B: analysis of Z(32BPA). C: analysis of Z(35BPA). For ESI-MS runs the proteins were diluted to approximately $50 \mathrm{ng} / \mu \mathrm{L}$ with pure water to reduce the number of ions from their 
storage buffer (1x PBS). Subsequently they were analyzed on a LC-ESI-MS system (a Thermo Ultimate3000 coupled with a Bruker Impact II instrument) using a ProSwift RP-4H column (product no. 069477 , Thermo and a gradient of solvent B ( $95 \%$ acetonitrile, $0.1 \%$ formic acid in water) against solvent $A$ ( $3 \%$ acetonitrile, $0.1 \%$ formic acid in water). The gradient was run at $0.4 \mathrm{~mL} / \mathrm{min}$ and started with 2 minutes of $4 \%$ solvent B, then raised from $4 \%$ to $90 \%$ B within 6 minutes followed by 2 minutes at $90 \%$ B and an equilibration step for the next run with $4 \%$ B for 4 minutes. All samples were ionized using electrospray in positive mode and detected with a mass range of 5000 to $50000 \mathrm{~m} / \mathrm{z}$ using a spectra rate of $1 \mathrm{~Hz}$.

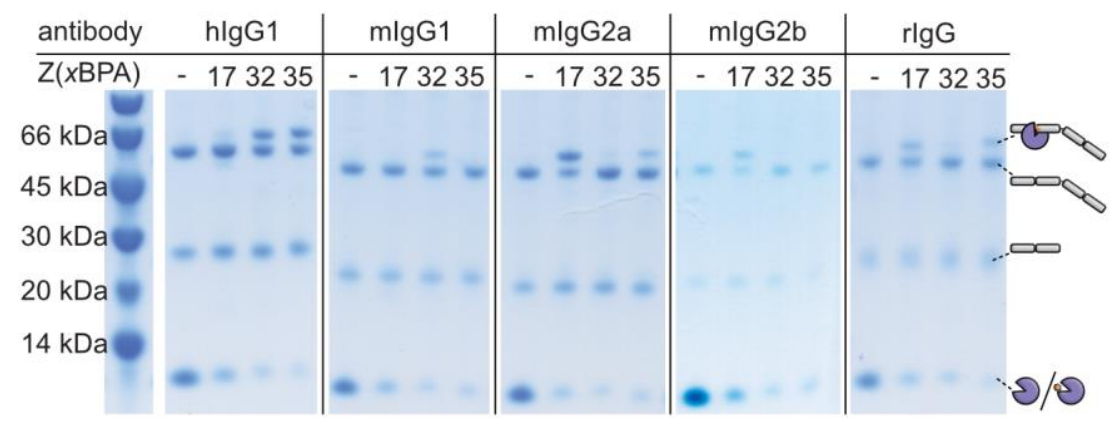

Figure S2: Labeling of all investigated antibodies with all Z(xBPA)-domains. The reactions were performed with conditions previously identified as optimal with small adjustments to our small scale screening. ${ }^{1-2}$ Briefly in a total volume of $5 \mu \mathrm{L}$ PBS an antibody concentration of $2 \mu \mathrm{M}$ and a Z(xBPA)-domain concentration of $10 \mu \mathrm{M}(5 \mathrm{eq})$ were provided and incubated under 365 nm UV light for 2 hours while chilled on ice.

Table S1: Comparision of published and measured conversions of heavy chains in photoaffinity labeling reactions with Z(xBPA)domains based on colorimetric calculation of reducing SDS-PAGEs stained with Coomassie Blue. Our reactions were performed with conditions as closely as possible to the previously published study. ${ }^{1}$ Briefly in a total volume of 5 uL PBS an antibody concentration of $2 \mathrm{uM}$ and a Z(xBPA)-domain concentration of $10 \mathrm{uM}$ ( 5 eq) were provided and incubated under $365 \mathrm{~nm}$ UV light for 2 hours while chilled on ice. Analysis was performed on at least three experiments performed on different days and with different antibodies, if available.

\begin{tabular}{|c|c|c|c|c|c|}
\hline \multicolumn{2}{|c|}{ IgG } & \multirow{2}{*}{$Z(x B P A)$} & \multicolumn{2}{|c|}{ conversion of heavy chains (\%) } & \multirow[t]{2}{*}{ Z-domain / antibody } \\
\hline species & subtype & & published $^{1}$ & measured & \\
\hline \multirow{3}{*}{ human } & \multirow{3}{*}{$\operatorname{lgG1}$} & 17 & little & $9 \pm 1$ & $0.2 \pm 0.02$ \\
\hline & & 32 & 37 & $37 \pm 12$ & $0.7 \pm 0.24$ \\
\hline & & 35 & 47 & $45 \pm 7$ & $0.9 \pm 0.14$ \\
\hline \multirow{9}{*}{ mouse } & \multirow{3}{*}{$\operatorname{lgG1}$} & 17 & none & 0 & 0.0 \\
\hline & & 32 & 50 & $25 \pm 3$ & $0.5 \pm 0.06$ \\
\hline & & 35 & none & 0 & 0.0 \\
\hline & \multirow{3}{*}{$\lg G 2 a$} & 17 & 60 & $55 \pm 7$ & $1.1 \pm 0.14$ \\
\hline & & 32 & little & 0 & 0.0 \\
\hline & & 35 & modestly & 23 & 0.5 \\
\hline & \multirow{3}{*}{$\operatorname{lgG} 2 \mathrm{~b}$} & 17 & 71 & $50 \pm 3$ & $1.0 \pm 0.06$ \\
\hline & & 32 & none & 0 & 0.0 \\
\hline & & 35 & none & 0 & 0.0 \\
\hline \multirow{3}{*}{ rabbit } & \multirow{3}{*}{$\lg G$} & 17 & 30 & $43 \pm 9$ & $0.9 \pm 0.18$ \\
\hline & & 32 & none & $14 \pm 6$ & $0.3 \pm 0.12$ \\
\hline & & 35 & 34 & $36 \pm 7$ & $0.7 \pm 0.14$ \\
\hline
\end{tabular}


A
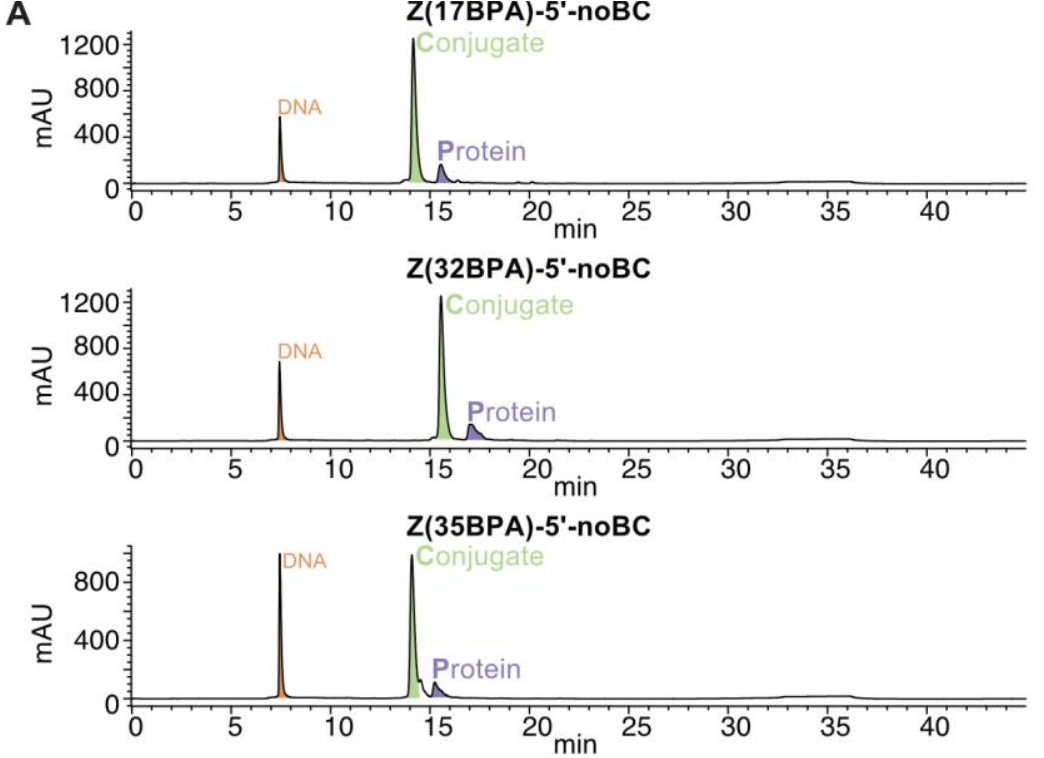

B

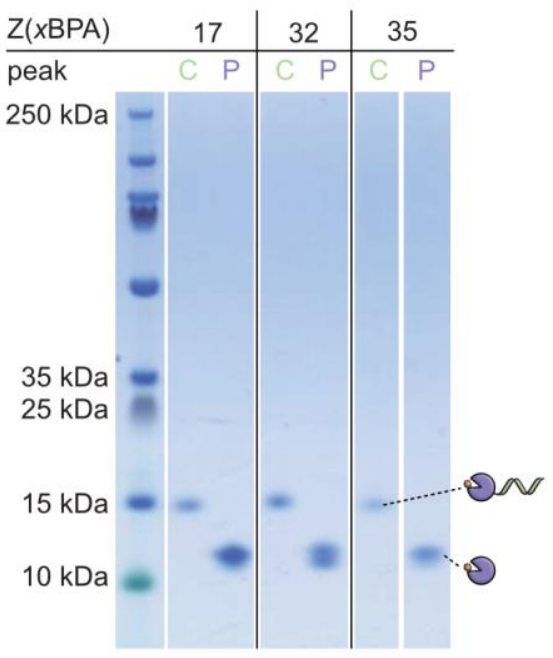

Figure S3: Exemplary purification of Z(xBPA)-5'-noBC-conjugates. A: IP-RP-HPLC chromatograms at $260 \mathrm{~nm}$ for conjugates of Z(xBPA)-5'-noBC on a Zorbax 300SB-C18 column $(3.5 \mu \mathrm{m}, 4.6 \times 150 \mathrm{~mm}$, Agilent) using a gradient of 2.5 minutes at $0 \%$ solvent $B$, $0-20 \%$ B in 2.5 minutes, $20-40 \%$ B in 20 minutes, $40-95 \%$ B in 2.5 minutes, $95 \%$ B for 5 minutes, $95-20 \%$ B in 2.5 minutes, $0 \%$ B for 10 minutes (solvent $A: 25 \mathrm{mM}$ triethylammonium buffer $\left(\mathrm{pH} \mathrm{7.5)}\right.$ in $\mathrm{ddH}_{2} \mathrm{O}$; solvent $\mathrm{B}$ : acetonitrile) at $45^{\circ} \mathrm{C}$. Peaks identified as DNA and protein by comparison of retention times with educts are highlighted in orange and purple, respectively. The newly formed peaks around 14 minutes were identified as conjugates (green). Minor peaks close to the protein peaks are possible hydrolyzation products of the protein lacking the $\mathrm{H}_{6}$-tag. B: reducing SDS-PAGE of conjugate and protein peaks from A showing the same electrophoretic mobility as the conjugate and protein bands identified in figure 2 . Please note that conjugate and protein fractions were solved in different volumes. Therefore, the intensity of the bands does not match to the area under the curves in A.

Table S2: Conversion of $\mathrm{G}_{3}$-modified DNA-oligonucleotides in Sortase A mediated DNA-modification of proteins as determined via SDS-PAGE stained with GelRed and yield of Z(xBPA)-DNA-conjugates after ion-pairing reversed-phase HPLC purification. For reactions with $A B C$-carrying DNA-oligonucleotides the conversion was not determined via SDS-PAGE (n.d.).

\begin{tabular}{l|l|l|l}
$Z(x B P A)$ & DNA & conversion measured via SDS-PAGE (\%) & yield after IP RP-HPLC (\%) \\
\hline 17 & $5^{\prime}-$-noBC & 62 & $62 \pm 7$ \\
\hline & $5^{\prime}-A B C 1$ & n.d. & 47 \\
\hline & $5^{\prime}-A B C 2$ & n.d. & 49 \\
\hline & $5^{\prime}-A B C 3$ & n.d. & 44 \\
\hline & $3^{\prime}-$ noBC & 69 & 72 \\
\hline & $3^{\prime}-A B C 1$ & n.d. & 60 \\
\hline & $3^{\prime}-A B C 2$ & n.d. & 54 \\
\hline 32 & $3^{\prime}-A B C 3$ & n.d. & 62 \\
\hline & $5^{\prime}-$ noBC & 56 & $54 \pm 3$ \\
\hline 35 & $3^{\prime}-$ noBC & 64 & 67 \\
\hline & $5^{\prime}-$ noBC & 63 & $47 \pm 10$ \\
& $3^{\prime}-$ noBC & 65 & 53
\end{tabular}



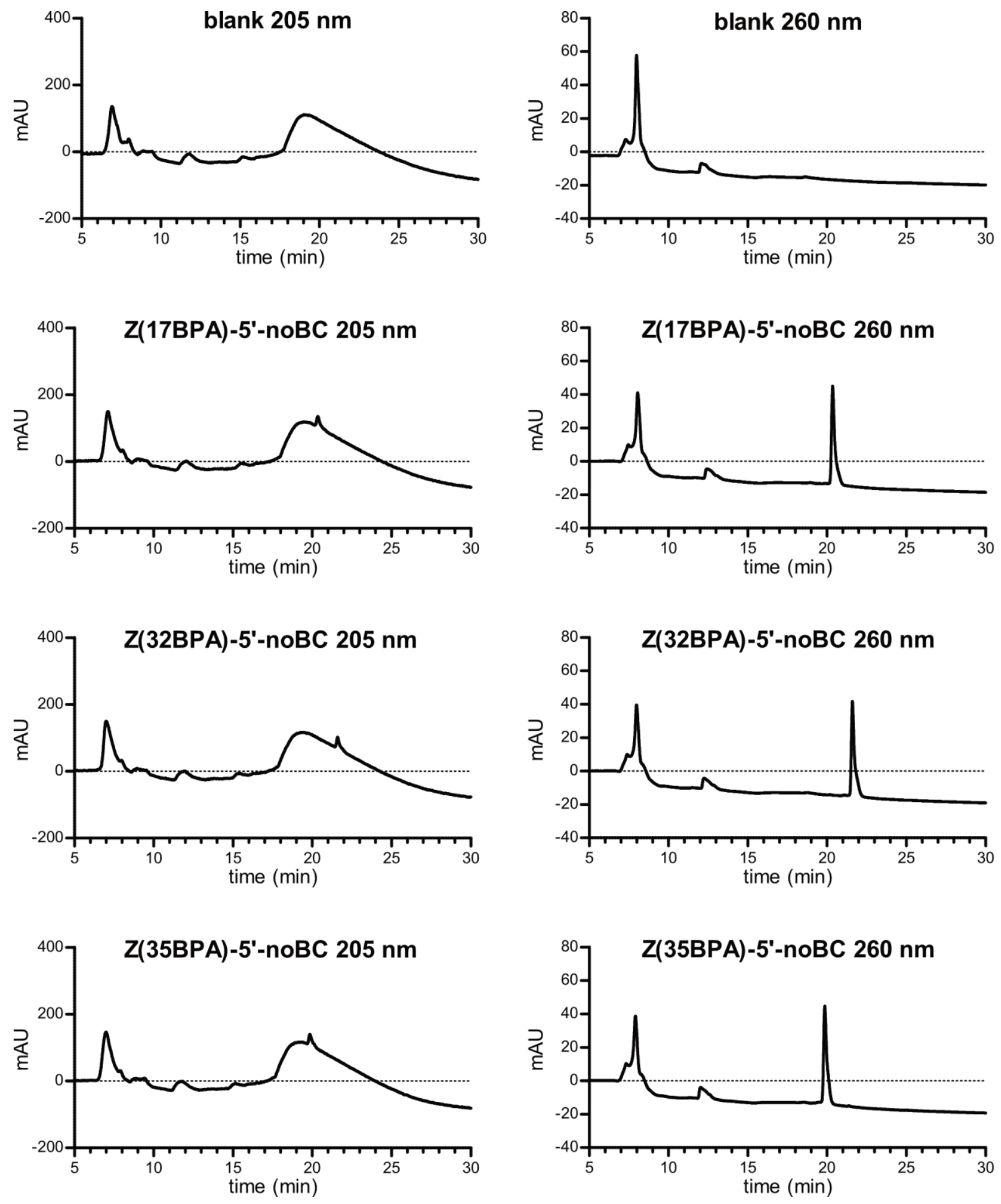

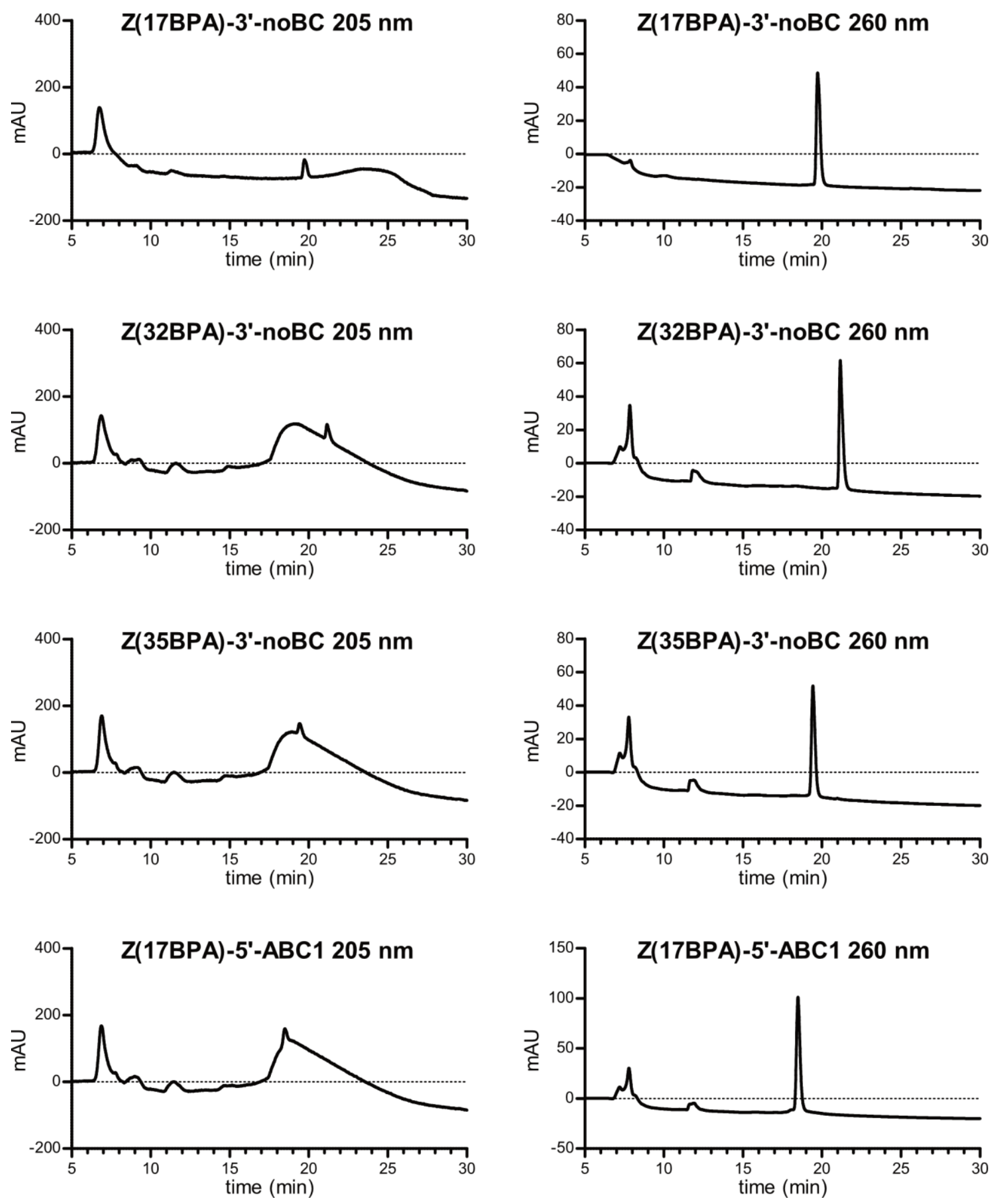

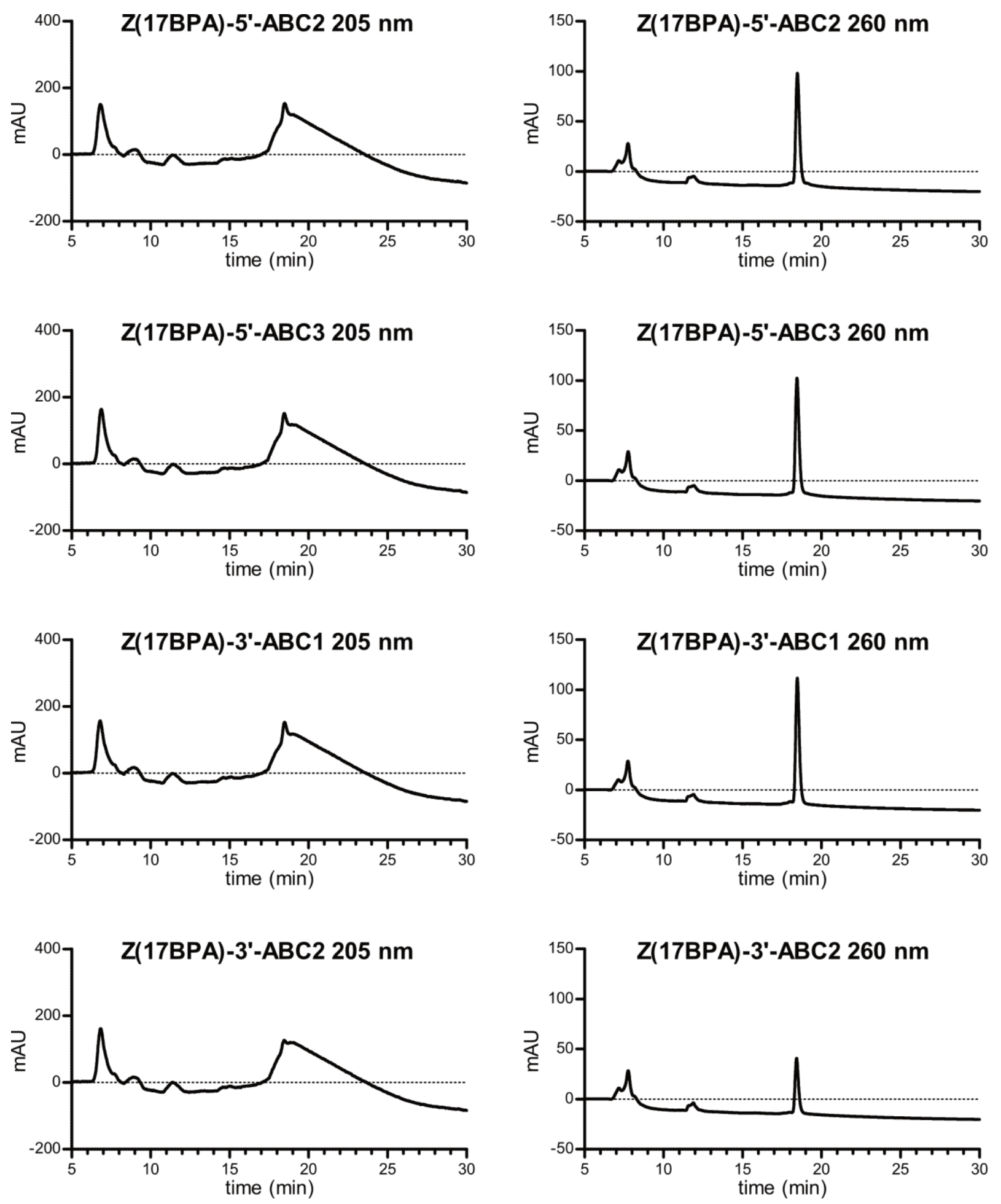

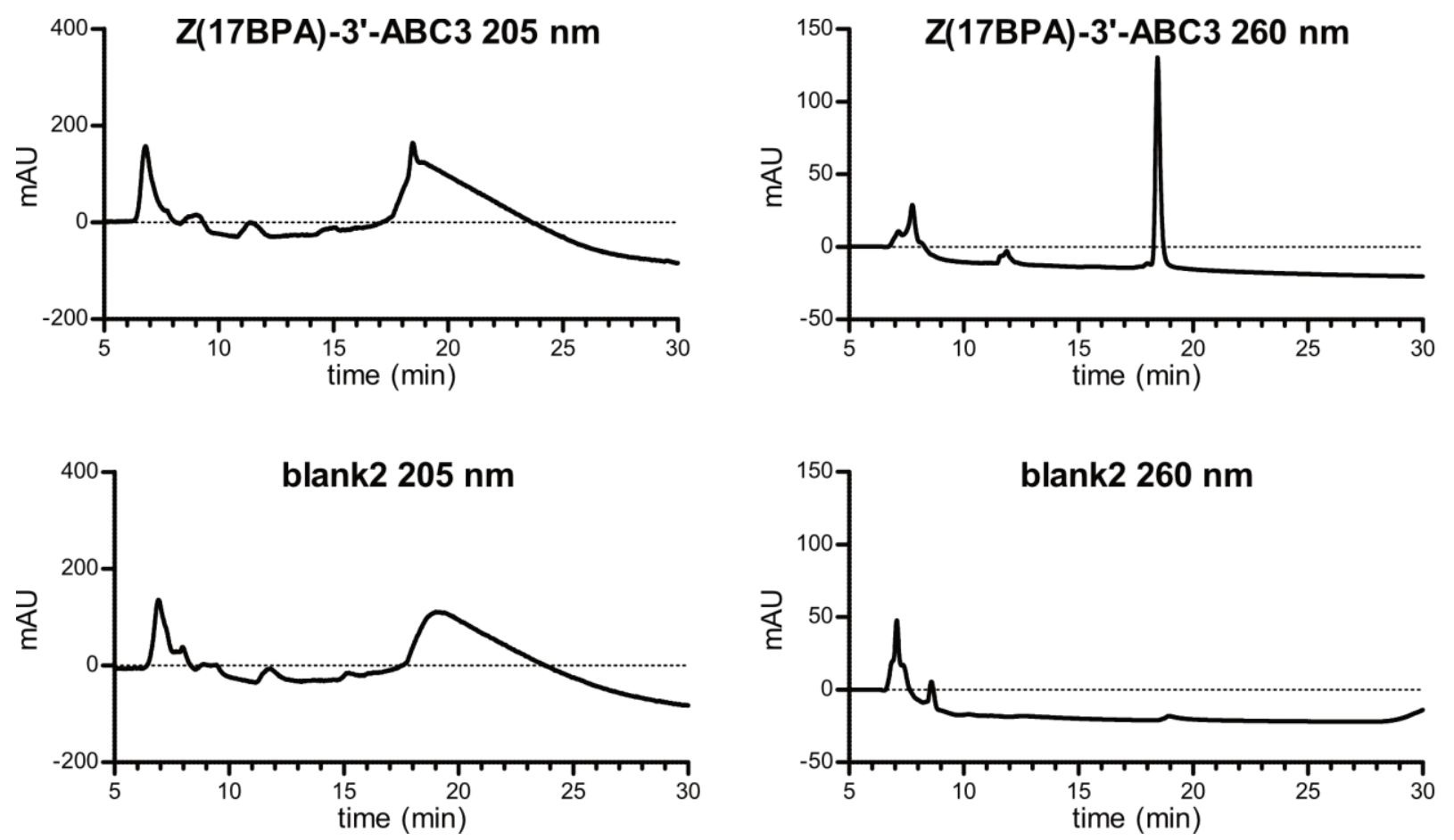

Figure S4: Analytical IP-RP-HPLC runs for Z(xBPA)-DNA-conjugates on a Zorbax 300SB-C18 column (solvent A: $25 \mathrm{mM}$ triethylammonium buffer $(\mathrm{pH} 7.5)$ in $\mathrm{dd} \mathrm{H}_{2} \mathrm{O}$; solvent $\mathrm{B}$ : acetonitrile; gradient: $0 \%$ B for $2.5 \mathrm{~min} ., 0-5 \% \mathrm{~B}$ in 2.5 min., $5-30 \% \mathrm{~B}$ in 25 min., 30-95\% B in $2.5 \mathrm{~min}$., $95 \%$ B for $5 \mathrm{~min}$., $95-0 \%$ B in $2.5 \mathrm{~min}$. and $0 \% \mathrm{~B}$ for $10 \mathrm{~min}$. at $45^{\circ} \mathrm{C}$ ). Detection is shown at $260 \mathrm{~nm}$ for the main gradient (5-30\% solvent B in 25 min.) in chronological order of the injections with exception for Z(17BPA)-3'-noBC, which had to be re-measured due to technical reasons. As can be seen from the blank injections at the top and bottom peaks at approximately 8 and 12 minutes at both wavelength as well as the big peak around 20 min at $205 \mathrm{~nm}$ are impurities stemming from the column or buffer effects.

Table S3: Oligonucleotides utilized for Sortase A mediated modification of the Z(xBPA)-domains. Bold: sequences for antibody barcodes (ABCs) carried by all antibody molecules against the same antigen. Italic: sequences for unique molecular identifiers (UMIs) used to separate DNA amplification copies from unique antibody-antigen interactions. Underlined: Universal handles on barcoded oligonucleotides, which are used for emPCR reactions performed in DBS-Pro.

\begin{tabular}{|c|c|c|}
\hline name & sequence & modification \\
\hline $3^{\prime}-$ noBC & agt ctg gat gta gtc & $3^{\prime}-G_{3}$ \\
\hline $5^{\prime}-$ noBC & agt ctg gat gta gtc & $5^{\prime}-G_{3}$ \\
\hline $3^{\prime}-A B C 1$ & cta aca gga ttc agg tag cgt ann nnn ntt ata tca cga caa gag & $3^{\prime}-\mathrm{G}_{3}$ \\
\hline $5^{\prime}-A B C 1$ & cta aca gga ttc agg tag cgt ann nnn ntt ata tca cga caa gag & $5^{\prime}-G_{3}$ \\
\hline $3^{\prime}-A B C 2$ & cta aca gga ttc agg taa tag cnn nnn ntt ata tca cga caa gag & $3^{\prime}-G_{3}$ \\
\hline $5^{\prime}-A B C 2$ & cta aca gga ttc agg taa tag cnn nnn ntt ata tca cga caa gag & $5^{\prime}-G_{3}$ \\
\hline $3^{\prime}-A B C 3$ & cta aca gga ttc agg tag tgc ann nnn ntt ata tca cga caa gag & $3^{\prime}-G_{3}$ \\
\hline $5^{\prime}-A B C 3$ & cta aca gga ttc agg tag tgc ann nnn ntt ata tca cga caa gag & $5^{\prime}-G_{3}$ \\
\hline
\end{tabular}




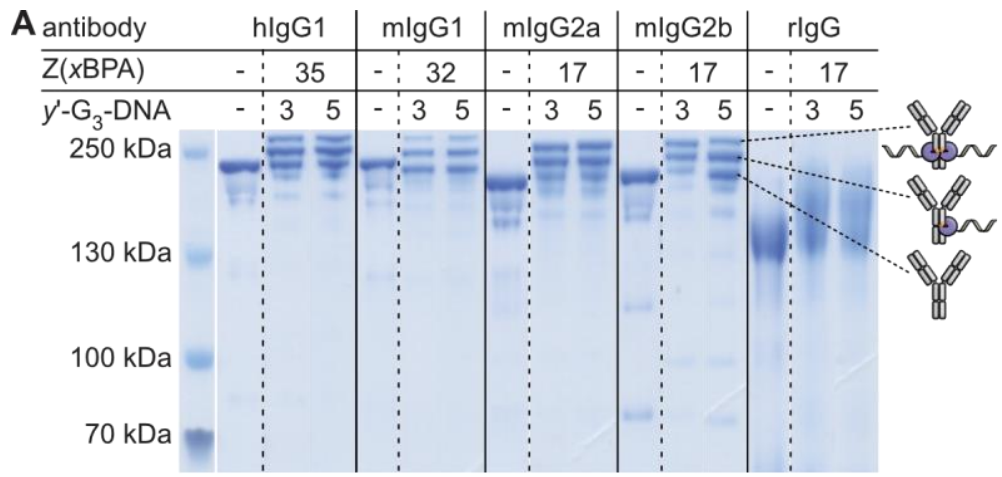

\begin{tabular}{|c|c|c|c|}
\hline B antibody & $\mathrm{Z}(x \mathrm{BPA})$ & $\mid y^{\prime}-G_{3}-n o B C$ & $\%$ conjugates \\
\hline \multirow[b]{2}{*}{ hlgG1 } & \multirow[b]{2}{*}{35} & 5 & $\begin{array}{l:l}41 & \text { di } \\
41 & 23\end{array}$ \\
\hline & & 3 & 23 \\
\hline \multirow{2}{*}{ mlgG1 } & \multirow{2}{*}{32} & 5 & 15 \\
\hline & & 3 & 12 \\
\hline \multirow{2}{*}{$\mathrm{mlgG} 2 \mathrm{a}$} & \multirow{6}{*}{17} & 5 & $41: 33$ \\
\hline & & 3 & 33 \\
\hline \multirow{2}{*}{$\mathrm{mlgG} 2 \mathrm{~b}$} & & 5 & 19 \\
\hline & & 3 & $41: 33$ \\
\hline \multirow{2}{*}{ rlgG } & & 5 & n.d. : n.d. \\
\hline & & 3 & $\begin{array}{l:l}\text { n.d. } & \text { n.d. }\end{array}$ \\
\hline
\end{tabular}

Figure S5: Analysis of photoaffinity labeling of representative antibodies from the subtypes human IgG1, mouse IgG1, mouse $\operatorname{lgG} 2 a$, mouse IgG2b and rabbit IgG using the best suited Z(xBPA)-domain for each IgG, respectively. Reaction solutions were prepared in $5 \mu \mathrm{L}$ scale containing $2 \mu \mathrm{M}$ of antibody and $10 \mu \mathrm{M}$ of the matching Z(xBPA)- $y^{\prime}$-noBC-conjugate (5 eq). UV conjugation was performed at $365 \mathrm{~nm}$ for $2 \mathrm{~h}$ on ice before the reactions were quenched by boiling with oxidizing SDS sample buffer. A: oxidizing SDS-PAGE. B: summary of percentages of mono- and di-modified antibody-DNA-conjugates for IgG subtypes shown in part A. Conjugate percentages are calculated from a single experiment.

Table S4: Summary of labeling efficiencies for polyclonal rabbit IgGs used for DBS-Pro. As all antibodies possessed different starting concentrations each reaction was performed under different conditions to obtain the highest number of DNA-barcodes per antibody possible. For each pair of antibody and DNA the final volume of the reaction, the final concentration of the antibody and the equivalents of the used Z(17BPA)-DNA conjugate as well as the conversion of heavy chains and the average number of DNA per antibody are stated. Conversion of heavy chains and average DNA number are calculated from a single SDS-PAGE experiment.

\begin{tabular}{l|l|l|l|l|l|l} 
antibody & $\begin{array}{c}\mathbf{V}_{\text {reaction }} \\
\text { (uL) }\end{array}$ & $\begin{array}{c}\mathbf{C}_{\text {antibody }} \\
\text { (uM) }\end{array}$ & DNA & $\begin{array}{c}\text { equivalents of } \\
\text { Z(17BPA)-DNA }\end{array}$ & $\begin{array}{c}\text { conversion of } \\
\text { heavy chains (\%) }\end{array}$ & $\begin{array}{c}\text { DNA / } \\
\text { antibody }\end{array}$ \\
\hline$a b 1$ & 65 & 0.1 & $3^{\prime}-A B C 1$ & 10 & 4 & 0.1 \\
\hline$a b 1$ & 65 & 0.1 & $5^{\prime}-A B C 1$ & 10 & 9 & 0.2 \\
\hline$a b 2$ & 13.2 & 0.5 & $3^{\prime}-A B C 2$ & 10 & 41 & 0.8 \\
\hline$a b 2$ & 13.2 & 0.5 & $5^{\prime}-A B C 2$ & 10 & 45 & 0.9 \\
\hline$a b 3$ & 6.7 & 1 & $3^{\prime}-A B C 3$ & 5 & 33 & 0.7 \\
\hline$a b 3$ & 6.7 & 1 & $5^{\prime}-A B C 3$ & 5 & 30 & 0.6
\end{tabular}


Table S5: Overview of libraries produced for DBS-Pro.

\begin{tabular}{c|cccc|c|c|c}
\multirow{2}{*}{ library } & \multicolumn{7}{|c}{ input of agx-y'-ABCx beads (\%) } \\
\cline { 2 - 9 } & $\mathrm{y}=$ & \multicolumn{3}{c}{5} & 5 & \multicolumn{3}{|c}{3} \\
& $\mathrm{x}=$ & 1 & 2 & 3 & 1 & 2 & 3 \\
\hline 1 & & 100 & 0 & 0 & 0 & 0 & 0 \\
\hline 2 & & 0 & 100 & 0 & 0 & 0 & 0 \\
\hline 3 & & 0 & 0 & 100 & 0 & 0 & 0 \\
\hline 4 & & 33 & 33 & 33 & 0 & 0 & 0 \\
\hline 5 & & 80 & 10 & 10 & 0 & 0 & 0 \\
\hline 6 & & 10 & 80 & 10 & 0 & 0 & 0 \\
\hline 7 & & 10 & 10 & 80 & 0 & 0 & 0 \\
\hline 8 & & 0 & 0 & 0 & 100 & 0 & 0 \\
\hline 9 & & 0 & 0 & 0 & 0 & 100 & 0 \\
\hline 10 & & 0 & 0 & 0 & 0 & 0 & 100
\end{tabular}

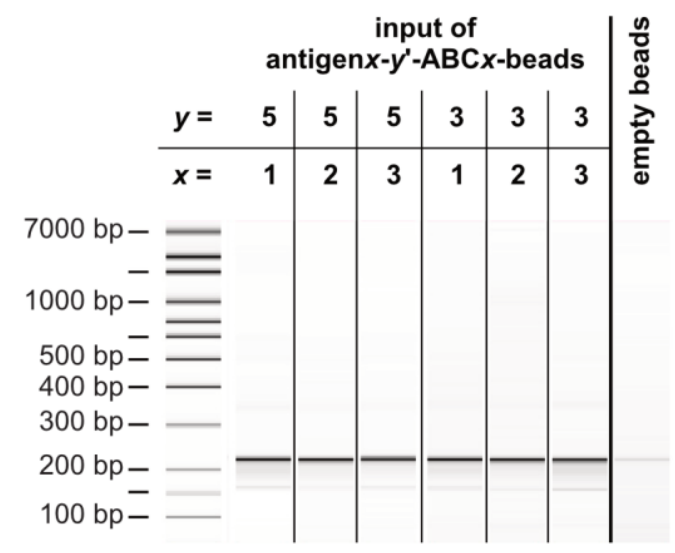

Figure S6: Data from representative Bioanalyzer runs during the DBS-Pro workflow. 


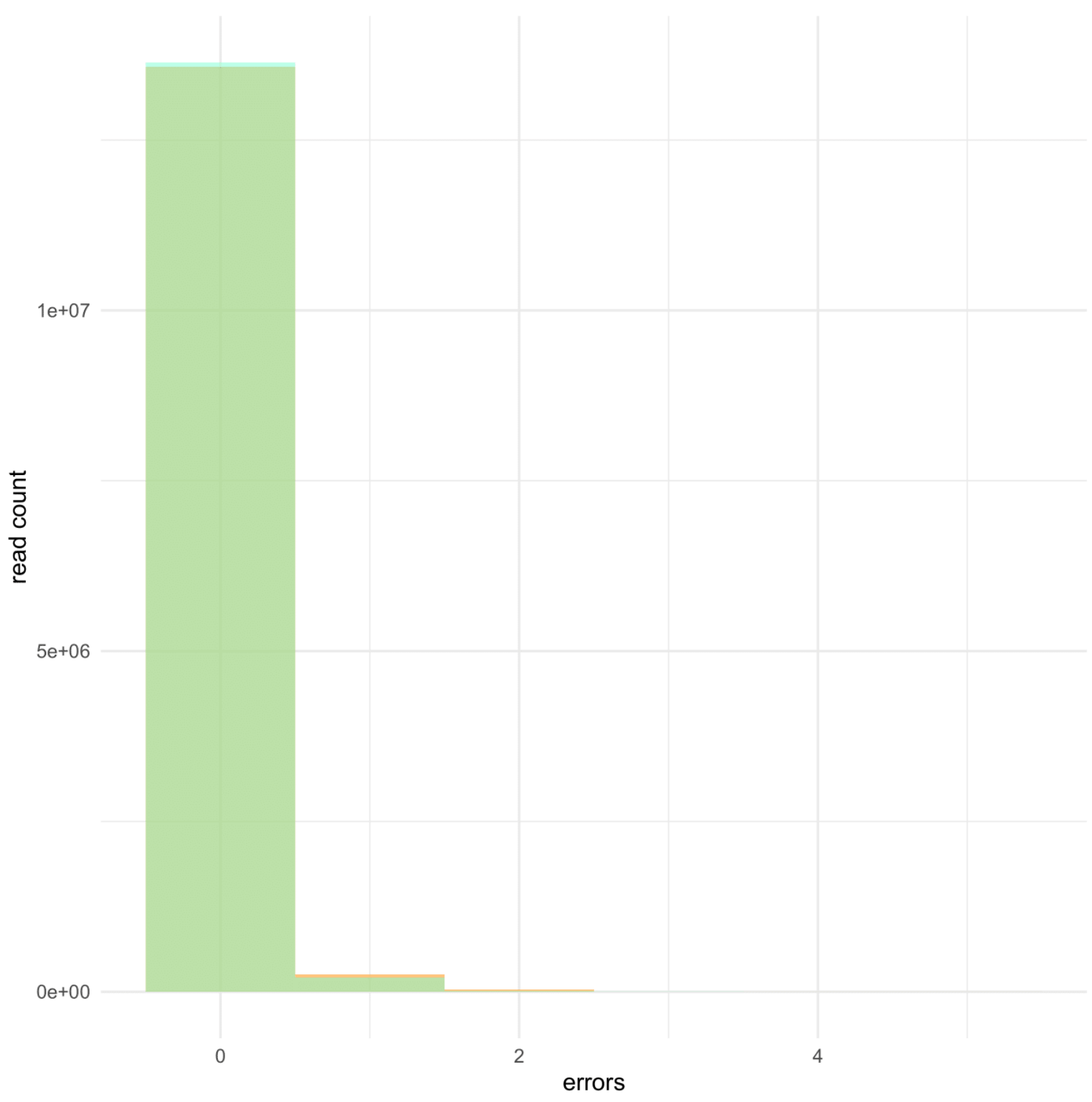

Figure S7: Error count histogram for DBS- (teal) and $A B C x$-sequences (orange) with their overlap marked in green. Errors were counted over all five $A B C x$ bases, while only the first five DBS bases were taken into account to correct for their different lengths. As the DBS sequences are based on randomized DNA-stretches, namely an NBDHV sequence in the first five nucleotides, only $1 / 3$ of the occurring errors can be detected. Therefore, DBS error counts were corrected by counting the number of DBS errors, multiplying it by 2 and randomly dispersing it over the data. 
Table S6: DBS-Pro UMI distribution. Library theoretical bead mixture (left) and DBS-Pro UMI percentiles (right) where the distribution of UMIs corresponds to the antigen bead distribution detected in the sample.

\begin{tabular}{c|c|c|c||c|c|c|c} 
& \multicolumn{2}{|c|}{ Theoretical mixture } & \multicolumn{4}{c}{ Detected percentiles } \\
\hline Library & PrEST 1 & PrEST 2 & PrEST 3 & PrEST 1 & PrEST 2 & PrEST 3 & Sum UMIs \\
\hline 1 & $100.00 \%$ & $0 \%$ & $0 \%$ & $99.99 \%$ & $0.00 \%$ & $0.01 \%$ & 974,299 \\
\hline 2 & $0 \%$ & $100.00 \%$ & $0 \%$ & $0.00 \%$ & $100.00 \%$ & $0.00 \%$ & 997,059 \\
\hline 3 & $0 \%$ & $0 \%$ & $100.00 \%$ & $0.00 \%$ & $0.00 \%$ & $100.00 \%$ & $1,296,055$ \\
\hline 4 & $33 \%$ & $33 \%$ & $33 \%$ & $23.76 \%$ & $46.45 \%$ & $29.78 \%$ & 885,012 \\
\hline 5 & $80 \%$ & $10 \%$ & $10 \%$ & $74.43 \%$ & $18.07 \%$ & $7.50 \%$ & 916,687 \\
\hline 6 & $10 \%$ & $80 \%$ & $10 \%$ & $4.20 \%$ & $91.31 \%$ & $4.49 \%$ & 805,920 \\
\hline 7 & $10 \%$ & $10 \%$ & $80 \%$ & $11.81 \%$ & $9.77 \%$ & $78.41 \%$ & 863,784
\end{tabular}

Table S7: Oligonucleotides utilized during the DBS-Pro workflow. Each DBS molecule contains universal handles (underlined) for amplification and a unique sequence used to separate read sequences into droplet-specific groups.

\begin{tabular}{|c|c|c|}
\hline name & sequence & used as \\
\hline $\mathrm{H} 1$ & gat atg acg atg cta at & \multirow{4}{*}{$\begin{array}{l}\text { primers } \\
\text { in emPCR }\end{array}$} \\
\hline $\mathrm{H} 3 \mathrm{R}-\mathrm{H} 2$ & ctg aat cct gtt aga tgg tct att gac tct $t$ & \\
\hline $\mathrm{H} 3 \mathrm{~F}$ & cta aca gga ttc agg ta & \\
\hline Bio_H4 & ctc ttg tcg tga tat aa & \\
\hline DBS & $\begin{array}{l}\text { gat atg acg atg cta atc aga tca bdv hbd vhb dvh bdv hbd vha aga gtc aat } \\
\text { aga cca t }\end{array}$ & $\begin{array}{l}\text { droplet } \\
\text { barcodes }\end{array}$ \\
\hline ¡5 & $\begin{array}{l}\text { aat gat acg gcg acc acc gag atc tac act ctt tcc cta cac gac gct ctt ccg atc } \\
\text { tcg atg cta atc aga tca }\end{array}$ & \multirow{2}{*}{$\begin{array}{l}\text { primers } \\
\text { in } \\
\text { indexing } \\
\text { qPCR }\end{array}$} \\
\hline i7-index & $\begin{array}{l}\text { caa gca gaa gac ggc ata cga gat (index) gtg act gga gtt cag acg tgt gct ctt } \\
\text { ccg atc ctc ttg tcg tga tat aa }\end{array}$ & \\
\hline
\end{tabular}

1. Hui, J. Z.; Tsourkas, A., Optimization of Photoactive Protein Z for Fast and Efficient Site-Specific Conjugation of Native IgG. Bioconjugate Chemistry 2014, 25 (9), 1709-1719.

2. Westerlund, K.; Vorobyeva, A.; Mitran, B.; Orlova, A.; Tolmachev, V.; Karlström, A. E.; Altai, M., Site-specific conjugation of recognition tags to trastuzumab for peptide nucleic acid-mediated radionuclide HER2 pretargeting. Biomaterials 2019, 203, 73-85. 